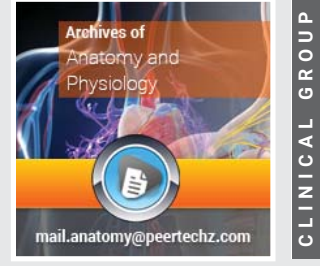

Opinion

\title{
Musculoskeletal system for healing of a bone fracture
}

\section{Padmavathi I*}

B.Sc Computers, Andhra University, India
Received: 15 December, 2019

Accepted: 26 December, 2019

Published: 28 December, 2019

*Corresponding author: Padmavathi I, B.Sc Computers, Andhra University, India, Tel: 9739432229; Email:padma25k@gmail.com https://www. peertechz.com

Check for updates
Fractures can be complete or partial. Some fractures needs surgery or metal plates, while some fractures may only need a brace. Types of fractures are transverse, oblique, spiral, and comminute, impacted, greenstick, open and closed. The healing of fractures begins with the formation of a hematoma by internal and external calli. A bone fracture will heal whether or not a physician resets it in its anatomical position. If the bone is reset incorrectly, the healing process will keep the bone in its deformed position. Everyone who experiences a fractured bone will heal differently. The healing process depends on the nature, the stability of fracture fixation, and biological processes, so a proper healing process is important. Bone healing or fracture healing is a proliferative physiological process in which the body facilitates the curing of a bone fracture.

In order to heal the fracture, the bones must be in the correct position and completely protected. After a fracture, the body acts to protect the injured area, and forms a protective blood clot and hard skin around the fracture. Some threads of bone cells grow on both sides of the fracture line. These threads grow toward each other. The fracture closes and the hard skin is absorbed.

Depends upon the severity of the fracture and how a person follows their doctor's recommendations, bones can take time to heal between weeks to several months, although it can vary depending on the type and site of the injury.

A closed reduction is manipulated and set into its natural position without surgery in the broken bone. Open reduction need a surgery to expose the fracture and reset the bone. While some fractures can be minor, others are quite severe and result in grave complications. For example, a fractured diaphysis of the femur has the potential to release fat globules into the bloodstream. These can block in the capillary beds of the lungs, leading to respiratory injured and if not treated quickly, death. Symptoms of a fracture that is not healing normally include swelling and a severe pain that may be felt deep within the affected bone. Repeatedly, the bone isn't strong enough to bear weight, and you may not be able to use the affected part of the body up to the bone heals.

Generally bone fracture treatment consists of a doctor reducing (pushing) dislocated bones back into place via replacement with or without anaesthetic, stabilizing their position to help and then waiting for the bone's natural healing process to occur.Sufficient nutrient intake has been found to ominously affect the integrity of the fracture healing. Age, bone type, drug analysis and pre-existent bone pathology are factors which affect healing. The role of bone healing is to produce new bone without a scar as seen in other muscles which would be a structural weakness or abnormality. The entire regeneration of the bone process can depends on the dislocation or fracture. But the bone formation generally spans the entire duration of the healing process, in some cases, bone marrow within the fracture has healed two or less weeks before the final adjusting part.

In view with the above, we can conclude that the fast and better healing of a fracture depends on few factors like, proper and regular treatment, type of fracture, bone strength and age. 